\title{
Blood pressure variability in relation to outcome in the International Database of Ambulatory blood pressure in relation to Cardiovascular Outcome
}

\author{
Katarzyna Stolarz-Skrzypek ${ }^{1,2}$, Lutgarde Thijs ${ }^{1}$, Tom Richart ${ }^{1,3}$, Yan Li ${ }^{4,5}$, Tine W Hansen ${ }^{6}$, José Boggia ${ }^{7}$, \\ Tatiana Kuznetsova ${ }^{1}$, Masahiro Kikuya ${ }^{8}$, Kalina Kawecka-Jaszcz ${ }^{2}$ and Jan A Staessen ${ }^{1,3}$
}

Ambulatory blood pressure (BP) monitoring provides information not only on the BP level but also on the diurnal changes in BP. In the present review, we summarized the main findings of the International Database on Ambulatory BP in relation to Cardiovascular Outcome (IDACO) with regard to risk stratification based on BP variability. The predictive accuracy of daytime and nighttime BP and the night-to-day BP ratio depended on the disease outcome under study and treatment status, and differed for fatal outcomes compared with the composite of fatal and nonfatal diseases. An exaggerated morning surge, exceeding the 90th percentile of the population, is an independent risk factor for mortality and cardiovascular and cardiac events. Conversely, a sleep-trough or preawakening morning surge in systolic BP below $20 \mathrm{~mm} \mathrm{Hg}$ is probably not associated with an increased risk of death or cardiovascular events. BP variability as captured by the average of the daytime and nighttime s.d. weighted for the duration of the daytime and nighttime interval (s.d.dn) and the average real variability $\left(A_{R V} V_{24}\right)$ predicted the outcome, but improved the prediction of the composite of all cardiovascular events by only $0.1 \%$. In conclusion, the IDACO observations support the concept that BP variability adds to risk stratification, but above all highlight that 24-h ambulatory BP level remains the main predictor to be considered in clinical practice.

Hypertension Research (2010) 33, 757-766; doi:10.1038/hr.2010.110; published online 8 July 2010

Keywords: blood pressure; cardiovascular outcome; diurnal blood pressure variability; morning surge

\section{INTRODUCTION}

Ambulatory monitoring allows the registration of blood pressure (BP) throughout the day in patients engaged in their usual activities. Ambulatory BP recordings have high reproducibility, are not subject to digit preference, and avoid the transient rise of a patient's BP in response to a medical environment, the so-called white-coat effect. ${ }^{1,2}$ Collaborative meta-analyses of individual patient data constitute a powerful research tool to clarify the role of cardiovascular risk factors in relation to total and cause-specific mortality and morbidity, over and beyond the prognostic information generated by single-cohort studies. $^{3,4}$ The international database on ambulatory BP monitoring ${ }^{5}$ illustrated to what extent a meta-analysis of individual patient data can contribute to our understanding of the distribution ${ }^{5,6}$ and the diurnal profile $e^{5,7}$ of ambulatory BP across ethnically diverse populations. This database, constructed in $1993-1994,{ }^{5}$ however, lacked a prospective dimension. We therefore planned to build a shared new resource of prospective studies conducted in the general population with the objective of elucidating with great precision to what extent ambulatory BP improves risk stratification over and beyond conventional BP. We chose IDACO as the acronym for the new International Database of Ambulatory BP in relation to Cardiovascular Outcome. Studies were eligible for inclusion if they comprised a random population sample, if information on the conventional and ambulatory BP levels were available at baseline, and if subsequent follow-up included fatal and nonfatal outcomes. All studies included in the IDACO received ethics approval and have been previously published in peer-reviewed journals. ${ }^{6,8-13}$ All participants provided informed written consent. In this review, we focused on BP variability, summarized the state-of-the art before IDACO and also highlighted what IDACO added to current knowledge.

${ }^{1}$ Studies Coordinating Centre, Division of Hypertension and Cardiovascular Rehabilitation, Department of Cardiovascular Diseases, University of Leuven, Leuven, Belgium; ${ }^{2}$ First Department of Cardiology and Hypertension, Jagiellonian University Medical College, Kraków, Poland; ${ }^{3}$ Department of Epidemiology, Maastricht University, Maastricht, The Netherlands; ${ }^{4}$ Center for Epidemiological Studies and Clinical Trials, Shanghai Jiaotong University School of Medicine, Shanghai, China; ${ }^{5}$ Center for Vascular Evaluation, Ruijin Hospital, Shanghai Jiaotong University School of Medicine, Shanghai, China; ${ }^{6}$ Department of Clinical Physiology, Nuclear Medicine and PET, Copenhagen University Hospital, Faculty of Health Sciences, Rigshospitalet, Copenhagen, Denmark; ${ }^{7}$ Centro di Nefrología and Departamento de Fisiopatologia, Hospital de Clínicas, Universidad de la República, Montevideo, Uruguay and ${ }^{8}$ Tohoku University Graduate School of Pharmaceutical Sciences and Medicine, Tohoku University, Sendai, Japan

Correspondence: Dr JA Staessen, Studies Coordinating Centre, Laboratory of Hypertension, University of Leuven, Campus Sint Rafaël, Kapucijnenvoer 35, Block d, Level 00, Leuven BE-3000, Belgium.

E-mail: jan.staessen@med.kuleuven.be or ja.staessen@epid.unimaas.n

Received 22 March 2010; revised 19 May 2010; accepted 2 June 2010; published online 8 July 2010 


\section{DAY-TO-NIGHT BP VARIABILITY}

\section{State-of-the-art before IDACO}

In 1988, O'Brien et al. ${ }^{14}$ reported for the first time that an abnormal circadian BP profile with a less marked decrease in nighttime BP level led to an increased risk of stroke. Subsequent studies of populations ${ }^{8,12,15,16}$ and hypertensive cohorts ${ }^{17-23}$ generally corroborated that a raised nocturnal BP predicted a higher rate of cardiovascular complications. Despite the apparent agreement between these previously published large-scale studies, $8,12,15-23$ several potential limitations warranted further clarification of the prognostic accuracy of the day $v$ s. night ambulatory BP. Many studies considered only fatal outcomes $^{8,12,21,22}$ or did not have the power to study cause-specific cardiovascular end points. ${ }^{8,12,16,20}$ Investigators dichotomized the night-to-day BP ratio, and applied different definitions of dipping status or different daytime and nighttime intervals. Few reports formally compared the predictive value of the BP at night over and beyond the daytime value. Finally, in cohorts of patients with hypertension, antihypertensive drug treatment attenuated the association between outcome and BP. ${ }^{18}$

\section{Information added by IDACO}

We aimed to report risk estimates that were independently associated with the daytime and nighttime BP level. In addition, we investigated in categorical and continuous analyses whether the night-to-day BP ratio contained any prognostic information over and beyond the 24-h BP. ${ }^{24}$ Table 1 summarizes the number of participants included in this analysis by ethnicity and country and their baseline characteristics.

When adjusted for daytime BP, nighttime BP predicted the total and cardiovascular mortality (Table 2). With adjustments applied for cohort and risk factors, the daytime and nighttime systolic and diastolic BPs consistently predicted cardiovascular, cardiac and coronary events, and fatal and nonfatal stroke. In fully adjusted models, with correction for nighttime BP, the systolic daytime BP lost its prognostic significance for cardiac events, whereas the diastolic daytime BP became nonsignificant for cardiac and coronary events. When adjusted for daytime BP, the systolic and diastolic nighttime levels no longer predicted coronary events (Table 2). Antihypertensive drug treatment removed the significant association between cardiovascular events and daytime BP. As in the continuous analyses, the hazard ratios (HRs) associated with extreme, decreased and reverse dipping $v s$. normal dipping showed an increasing risk from extreme to reverse dipping for mortality, but the results were inconsistent when considering fatal combined with nonfatal events.

In continuous analyses, the systolic and diastolic night-to-day ratios significantly predicted total and cardiovascular mortality in models adjusted for cohort and risk factors. This finding was also noted in fully adjusted models, which were additionally adjusted for 24-h BP. The only exception was the fully adjusted association of cardiovascular mortality with the systolic night-to-day ratio (Table 2). In contrast with mortality, the systolic and diastolic night-to-day ratios were inconsistent predictors of fatal combined with nonfatal cardiovascular events. In fully adjusted models, the systolic night-to-day ratio was not significant for all combined fatal and nonfatal outcomes investigated. The diastolic night-to-day ratio was only a significant predictor of all cardiovascular outcomes, but not of other combined fatal and nonfatal events (Table 2).

Figure 1 shows Kaplan-Meier survival function estimates for total mortality and the composite cardiovascular end point by category of the systolic night-to-day ratio. With adjustments applied for cohort, cardiovascular risk factors and the 24-h BP, participants with higher nighttime than daytime BP or with a nondipping nocturnal fall had
Table 1 Characteristics of the study groups included in the IDACO blood pressure variability analyses

\begin{tabular}{|c|c|c|c|}
\hline Label & $\begin{array}{l}\text { Day-to-night } \\
\text { variability }^{24}\end{array}$ & $\begin{array}{l}\text { Morning } \\
\text { surge }^{35}\end{array}$ & $\begin{array}{l}\text { Short-term } \\
\text { variability }^{49}\end{array}$ \\
\hline Total number available & 9828 & 9488 & 11785 \\
\hline \multicolumn{4}{|l|}{ Exclusion criteria } \\
\hline Age $<18$ years old at enrollment & 15 & 250 & 252 \\
\hline Conventional blood pressure not available & 217 & & \\
\hline$<10$ daytime or $<5$ nighttime recordings & 2138 & 2430 & 1892 \\
\hline$<2$ blood pressure readings before awaking & & 122 & \\
\hline$<2$ blood pressure readings after awaking & & 1041 & \\
\hline \multicolumn{4}{|l|}{ Missing readings during } \\
\hline 3 consecutive hours & & & 703 \\
\hline \multicolumn{4}{|l|}{ Number analyzed per ethnicity and country } \\
\hline \multicolumn{4}{|l|}{ Europe } \\
\hline Copenhagen, Denmark ${ }^{8}$ & 2137 & 1685 & 2018 \\
\hline Noorderkempen, Belgium ${ }^{9}$ & 1124 & 532 & 1086 \\
\hline Uppsala, Sweden 15 & 1097 & - & 1069 \\
\hline Novosibirsk, Russia ${ }^{10}$ & - & 220 & 226 \\
\hline Padova, Italy ${ }^{11}$ & - & 290 & 303 \\
\hline Kraków, Poland ${ }^{11}$ & - & 296 & 306 \\
\hline Pilsen, The Czech Republic ${ }^{11}$ & - & - & 161 \\
\hline Dublin, Ireland ${ }^{55}$ & - & - & 900 \\
\hline \multicolumn{4}{|l|}{ Asia } \\
\hline Ohasama, Japan ${ }^{12}$ & 1317 & 1396 & 1430 \\
\hline JingNing county, China ${ }^{6}$ & 349 & 327 & 346 \\
\hline \multicolumn{4}{|l|}{ South America } \\
\hline Montevideo, Uruguay ${ }^{13}$ & 1434 & 899 & 1093 \\
\hline \multirow[t]{2}{*}{ Age, years (s.d.) } & 56.8 & 53.0 & 53.0 \\
\hline & $(13.9)$ & $(14.7)$ & $(15.8)$ \\
\hline \multirow[t]{2}{*}{ Women, $n(\%)$} & 3416 & 3048 & 4785 \\
\hline & $(46 \%)$ & $(54 \%)$ & $(47 \%)$ \\
\hline \multirow[t]{2}{*}{ Hypertension, $n$ (\%) } & 3436 & 2305 & 3664 \\
\hline & $(46 \%)$ & $(41 \%)$ & $(41 \%)$ \\
\hline Antihypertensive treatment, $n$ & 1637 & 1188 & 1749 \\
\hline (\% of hypertensives) & $(48 \%)$ & $(52 \%)$ & $(48 \%)$ \\
\hline Median follow-up, years & 9.6 & 11.4 & 11.3 \\
\hline Deaths, $n$ & 983 & 785 & 1242 \\
\hline Cardiovascular complications, $n$ & 943 & 611 & 1049 \\
\hline
\end{tabular}

Abbreviation: IDACO, International Database on Ambulatory blood pressure in relation to Cardiovascular Outcome.

An ellipsis indicates that the analysis did not include this cohort.

higher rates of total mortality and cardiovascular events than those whose night-to-day BP ratio was normal or extreme $(P<0.0001)$.

\section{Interpretation}

In the IDACO database, the predictive accuracy of daytime and nighttime BP and the night-to-day BP ratio depended on the disease outcome under study and differed for fatal outcomes compared with the composite of fatal and nonfatal diseases (Figure 1). For fatal end points, nighttime BP performed better than the daytime BP, and the night-to-day BP ratio predicted mortality. In contrast, for fatal combined with nonfatal outcomes, the daytime BP performed equally well as the nighttime BP and the night-to-day BP ratio lost its prognostic accuracy. 
Table 2 Adjusted standardized hazard ratios for mortality and for combined fatal and nonfatal cardiovascular end points

\begin{tabular}{|c|c|c|c|c|c|c|}
\hline Label & Total mortality & Cardiovascular mortality & All cardiovascular events & Stroke & Cardiac & Coronary \\
\hline Systolic & $0.94(0.87-1.03)$ & $1.11(0.98-1.27)$ & $1.16(1.07-1.26)^{\ddagger}$ & $1.27(1.13-1.43)^{\ddagger}$ & $1.11(0.99-1.24)$ & $1.19(1.04-1.36)^{*}$ \\
\hline Diastolic & $0.94(0.87-1.03)$ & $1.07(0.94-1.22)$ & $1.11(1.02-1.20)^{*}$ & $1.21(1.07-1.37)^{\dagger}$ & $1.04(0.93-1.17)$ & $1.11(0.98-1.27)$ \\
\hline \multicolumn{7}{|l|}{ Nighttime blood pressure } \\
\hline Diastolic & $1.20(1.11-1.30)^{\S}$ & $1.24(1.10-1.40)^{\ddagger}$ & $1.20(1.11-1.30)^{\S}$ & $1.24(1.10-1.39)^{\ddagger}$ & $1.15(1.04-1.28)^{\dagger}$ & $1.08(0.96-1.22)$ \\
\hline \multicolumn{7}{|c|}{ Night-to-day blood pressure ratio } \\
\hline Systolic & $1.13(1.07-1.19)^{\S}$ & $1.08(0.99-1.17)$ & $1.05(0.98-1.11)$ & $1.02(0.94-1.11)$ & $1.05(0.97-1.14)$ & $0.97(0.89-1.07)$ \\
\hline Diastolic & $1.12(1.06-1.19)^{\ddagger}$ & $1.10(1.00-1.21)^{*}$ & $1.07(1.00-1.13)^{*}$ & $1.04(0.95-1.14)$ & $1.07(0.98-1.16)$ & $1.00(0.91-1.11)$ \\
\hline
\end{tabular}

Values are standardized hazard ratios (95\% confidence intervals), which express the risk per s.d. increase in the blood pressure variables. Systolic or diastolic s.d.'s were $15.52 / 9.33$ and $15.53 /$ $9.25 \mathrm{~mm} \mathrm{Hg}$ for the day and night blood pressures and 0.08/0.09 for the night-to-day blood pressure ratio. The cause of death was unknown in 36 cases. All hazard ratios were adjusted for cohort, sex, age, body mass index, smoking and drinking, serum cholesterol, history of cardiovascular disease, diabetes mellitus and antihypertensive drug treatment. The daytime blood pressure was additionally adjusted for the nighttime blood pressure (and vice versa), and the night-to-day ratio was additionally adjusted for the 24-h blood pressure.

Significance of the hazard ratios: ${ }^{*} P<0.05,{ }^{\dagger} P<0.01,{ }^{\ddagger} P<0.001$ and ${ }^{\$} P<0.0001$. Reproduced with permission from Boggia et al. ${ }^{24}$

The IDACO findings suggest that some of the associations between outcome and nondipping might reflect reverse causality. Indeed, a less pronounced nocturnal fall in BP might be a marker of physical inactivity during daytime as a consequence of concurrent disease or might result from the intake of BP-lowering drugs during daytime, which lower BP during daytime but not at night. Furthermore, participants with higher nighttime than daytime BP were not only older at enrollment, but were also older when they died than were those with a normal night-to-day ratio $(78.2$ vs. 74.1 years; $P<0.0001)$. Both cardiovascular $(78.9$ vs. 75.9 years; $P=0.002)$ and noncardiovascular (77.5 vs. 73.6 years; $P=0.001$ ) mortality contributed to this finding. The worse prognosis for participants with higher nighttime than daytime BP was therefore not associated with shorter life expectancy.

\section{Conclusion}

The 24-h BP levels ${ }^{25}$ rather than the dipping pattern should continue to inform clinical decisions. Furthermore, the classification of patients according to the night-to-day BP ratio greatly depends on arbitrary criteria, is poorly reproducible, ${ }^{26,27}$ and has a different prognostic meaning according to the disease outcome under study, the prevailing 24-h BP level and treatment status. We would therefore recommend that in future publications any categorical representation of the nightto-day BP ratio is supported by continuous analyses adjusted for the 24-h BP and be stratified for treatment status.

\section{THE MORNING SURGE IN BP}

\section{State-of-the-art before IDACO}

Several studies have shown that the incidence of cardiovascular complications peaks in the morning. ${ }^{28,29}$ For instance, in the Multicenter Investigation of Limitation of Infarct Size Study ${ }^{28}$ and in the Thrombolysis in Myocardial Infarction Phase II Trial, ${ }^{29}$ the incidence of myocardial infarction was highest between 0600 and 1200 hours. BP also follows a circadian pattern, generally characterized by a fall during sleep and a sharp rise upon awakening. ${ }^{30}$ This observation gave rise to the hypothesis that an exaggerated morning surge of BP might predict the cardiovascular outcome. However, previous studies of populations $^{31}$ and hypertensive patients ${ }^{18,32,33}$ produced contradictory results, possibly because of the small number of events and the lack of statistical power. A further issue complicating the interpretation of previous studies was the varying definitions of the morning surge in $\mathrm{BP}^{34}$

\section{Information added by IDACO}

For the analysis of morning surge, ${ }^{35}$ we selected studies in which the participants completed a diary during ambulatory BP monitoring (Table 1). Kario's seminal paper, ${ }^{32}$ published in 2003, introduced the definitions of the sleep-trough and pre-awakening morning surge in $\mathrm{BP}$ as a method to study the risk associated with the rise in BP level on awakening. For analysis of the morning surge in BP, we determined the awake and asleep periods from the participants' diary cards. Participants were asked to record the time when they got up in the morning and went to bed at night. The sleep-trough morning surge was the difference between the morning pressure (the average BP during the $2 \mathrm{~h}$ after awakening) and the lowest nighttime BP (the average of the lowest pressure and the two readings immediately preceding and following the lowest value). ${ }^{32}$ The pre-awakening morning surge was the difference between the morning BP (the average BP during the $2 \mathrm{~h}$ after awakening) and the pre-awakening $\mathrm{BP}$ (the average BP during the $2 \mathrm{~h}$ before awakening). ${ }^{31,32}$

In all participants, the sleep-trough and pre-awakening morning surge in systolic BP averaged $20.7 \pm 12.9$ and $13.1 \pm 11.9 \mathrm{~mm} \mathrm{Hg}$, respectively. However, there were significant ethnic and sex differences in the morning BP surge. The mean values of the sleep-trough systolic morning surge in the top decile were smaller in women than in men among Europeans (40.9 vs. $43.2 \mathrm{~mm} \mathrm{Hg} ; P=0.003)$ and South Americans ( 38.8 vs. $41.5 \mathrm{~mm} \mathrm{Hg} ;=0.041$ ), whereas the opposite was the case among Asians $(52.4$ vs. $50.1 \mathrm{~mm} \mathrm{Hg} ; P=0.064)$. For the pre-awakening systolic morning surge, the differences between women and men showed similar trends: 26.8 vs. $29.2 \mathrm{~mm} \mathrm{Hg}$ ( $P=0.062), 22.5$ vs. $26.9 \mathrm{~mm} \mathrm{Hg}(P=0.059)$ and 30.6 vs. $27.4 \mathrm{~mm} \mathrm{Hg}$ $(P=0.13)$ in Europeans, South Americans and Asians, respectively. These differences explain why we applied ethnicity- and sex-specific deciles to study the predictive value of the morning BP surge. This also ensured an equal distribution of ethnicities and women and men across the deciles. With adjustments applied for cohort, sex, age, body mass index, the 24-h systolic BP, current smoking, use of alcohol, serum cholesterol, the presence of diabetes mellitus, a history 


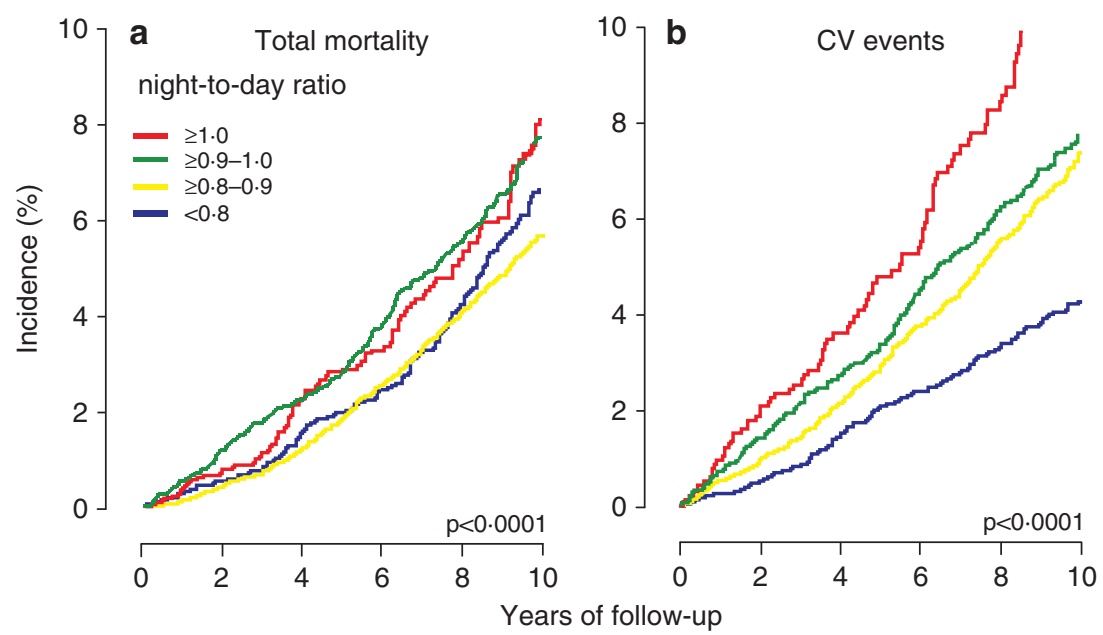

Figure 1 Kaplan-Meier survival function estimates for total mortality (a) and for all cardiovascular (CV) events (b) by category of the night-to-day ratio of systolic blood pressure. $P$-values are for trend across the four categories. Incidence was standardized to the distributions (mean or ratio) of cohort, sex, age, body mass index, smoking and drinking, serum total cholesterol, history of cardiovascular disease, diabetes mellitus and antihypertensive drug treatment. Reproduced with permission from Boggia et al. ${ }^{24}$

Table 3 Multivariable-adjusted hazard ratios for the sleep-trough and pre-awakening morning surge in systolic blood pressure

\begin{tabular}{|c|c|c|c|c|}
\hline \multirow[b]{2}{*}{ Outcomes (no. of events) } & \multicolumn{2}{|c|}{ Sleep-trough surge } & \multicolumn{2}{|c|}{ Pre-awakening surge } \\
\hline & Adjusted & Fully adjusted & Adjusted & Fully adjusted \\
\hline \multicolumn{5}{|l|}{ Mortality } \\
\hline All causes $(785)$ & $1.18(0.99-1.42)$ & $1.32(1.09-1.59)^{\dagger}$ & $1.11(0.91-1.35)$ & $1.23(1.00-1.51)^{\mathrm{a}}$ \\
\hline Cardiovascular (287) & $1.06(0.78-1.43)$ & $1.18(0.87-1.61)$ & $1.08(0.78-1.51)$ & $1.22(0.87-1.71)$ \\
\hline Noncardiovascular (473) & $1.28(1.01-1.61)^{\mathrm{a}}$ & $1.42(1.11-1.80)^{\dagger}$ & $1.13(0.87-1.46)$ & $1.23(0.95-1.61)$ \\
\hline \multicolumn{5}{|l|}{ Fatal and nonfatal events } \\
\hline All cardiovascular (611) & $1.18(0.97-1.44)$ & $1.30(1.06-1.60)^{\mathrm{a}}$ & $1.31(1.06-1.61)^{\dagger}$ & $1.45(1.17-1.80)^{\ddagger}$ \\
\hline Cardiac (317) & $1.36(1.04-1.78)^{a}$ & $1.52(1.15-2.00)^{\dagger}$ & $1.52(1.14-2.01)^{\dagger}$ & $1.69(1.26-2.27)^{\ddagger}$ \\
\hline Coronary (228) & $1.35(0.98-1.85)$ & $1.45(1.04-2.03)^{\mathrm{a}}$ & $1.50(1.08-2.09)^{\mathrm{a}}$ & $1.64(1.16-2.49)^{\dagger}$ \\
\hline Cerebrovascular (281) & $0.89(0.65-1.23)$ & $0.95(0.68-1.32)$ & $1.04(0.75-1.44)$ & $1.13(0.81-1.58)$ \\
\hline Infarction (140) & $0.79(0.49-1.27)$ & $0.85(0.52-1.39)$ & $1.26(0.82-1.92)$ & $1.46(0.93-2.30)$ \\
\hline Hemorrhage (70) & $1.57(0.90-2.73)$ & $1.46(0.81-2.63)$ & $1.18(0.64-2.18)$ & $1.11(0.59-2.11)$ \\
\hline
\end{tabular}

of cardiovascular disease and antihypertensive treatment, the risk of noncardiovascular mortality $(P=0.04)$ and of all fatal combined with nonfatal cardiac events $(P=0.03)$ was significantly higher in the top decile of the sleep-trough morning surge compared with the average risk in the whole study population (Table 3). When additionally adjusted for the night-to-day ratio of systolic $\mathrm{BP}$, the risk of all-cause mortality was $32 \%(P=0.004)$ higher in the top decile of the sleep-trough morning surge $(\geqslant 37.0 \mathrm{~mm} \mathrm{Hg})$. For cardiovascular and noncardiovascular death, these estimates were $18 \%(P=0.30)$ and $42 \%(P=0.005)$, and for all cardiovascular, cardiac, coronary and cerebrovascular events, $30 \%(P=0.01), 52 \%$ $(P=0.004), 45 \%(P=0.03)$ and $-5 \%(P=0.74)$, respectively (Table 3$)$. Similar results were found when analyzing the pre-awakening morning surge.
In an attempt to define in a more precise manner the cut-off points for risk stratification in clinical practice, we explored the risk associated with all values of the sleep-trough and pre-awakening morning surge in systolic BP within the 5th to 95th percentile interval. The overall risk in the whole study population was used as reference (Figure 2). For the sleep-trough morning surge in systolic BP, the lower boundary of the $95 \%$ confidence interval (CI) of the risk function crossed the unity of the HR at 24.6 and $20.9 \mathrm{~mm} \mathrm{Hg}$ for total mortality and all cardiovascular events, respectively. For the preawakening morning surge in systolic BP these crossings occurred at 22.7 and $21.5 \mathrm{~mm} \mathrm{Hg}$, respectively. The results of these analyses suggest that for both measures of the morning surge in systolic BP a value below $20 \mathrm{~mm} \mathrm{Hg}$ is probably not associated with increased risk (Figure 2). 

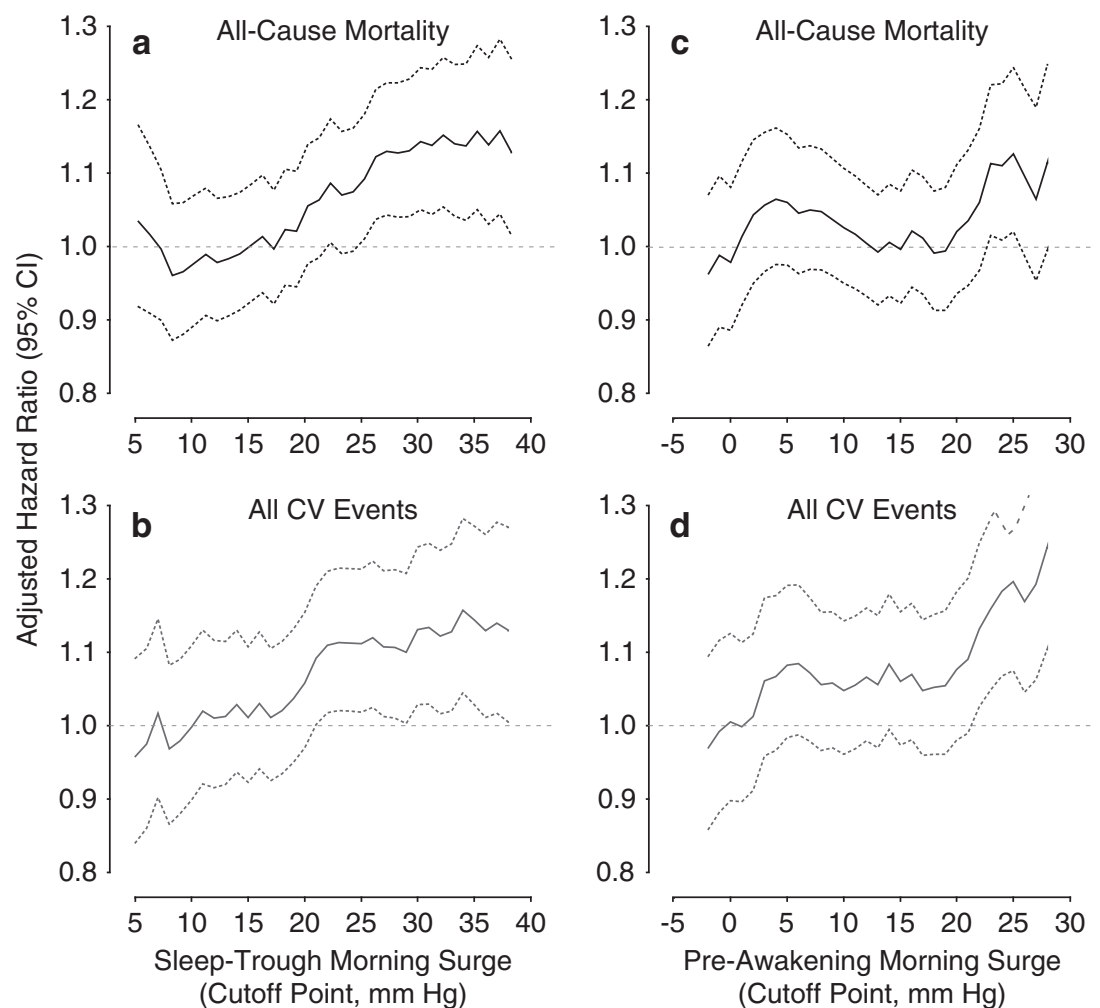

(Cutoff Point, $\mathrm{mm} \mathrm{Hg}$ )

Figure 2 Multivariable-adjusted HRs (solid lines) and their 95\% confidence intervals (dashed lines) for all-cause mortality (a, c) and for all fatal combined with nonfatal cardiovascular (CV) events (b, d) by cut-off points ranging from the 5th to 95th percentile for the sleep-trough (a, b) and pre-awakening (c, d) morning surge in systolic blood pressure (BP) in 5645 participants. The HRs express the risk in participants whose morning surge exceed the cut-off point compared with the average risk in the whole study population and were adjusted for cohort, sex, age, body mass index, smoking and drinking, serum cholesterol, history of cardiovascular disease, diabetes mellitus, antihypertensive drug treatment, 24-h systolic BP and the systolic night-to-day BP ratio. Reproduced with permission from Li et al. ${ }^{35}$

\section{Interpretation}

In 519 older hypertensive patients (mean age, 72 years) followed up for 41 months, Kario et al. ${ }^{32}$ compared the risk of silent and clinical cerebrovascular diseases in the top decile $(\geqslant 55 \mathrm{~mm} \mathrm{Hg}$ ) of the systolic sleep-trough morning surge with the risk in the other patients. After matching for age and the 24-h BP, the risk of multiple brain infarcts was approximately two fold higher in patients belonging to the top decile of the systolic sleep-trough morning surge. Moreover, with adjustment for the 24-h BP, nocturnal dipping status and the prevalence of silent infarcts at enrollment, the morning surge remained a significant predictor of stroke. ${ }^{32}$

In 1430 participants (mean age, 61 years; $64 \%$ women) randomly recruited from the Ohasama population, 128 cerebrovascular events occurred during a mean follow-up of 10.4 years. ${ }^{31}$ The cerebrovascular complications included 86 ischemic strokes and 27 cases with intracerebral hemorrhage. With adjustments applied for the 24-h systolic $\mathrm{BP}$ and other cardiovascular risk factors, the pre-awakening morning surge in systolic pressure tended to be associated with an increased risk of cerebral hemorrhage (HR per 1 s.d. increase, 1.34; 95\% CI, $0.95-1.89)$, but not with the risk of ischemic stroke $(0.97,95 \% \mathrm{CI}$, $0.79-1.19)$. The predictive value of the sleep-trough morning surge was broadly similar to that of the pre-awakening morning surge. ${ }^{31}$

Gosse et al. ${ }^{33}$ studied 507 untreated hypertensive patients without complications at enrollment. The average follow-up period of these patients was 92 months, during which 31 cardiovascular events, including 6 deaths, occurred. The morning surge was the difference between the first systolic measurement after standing up in the morning and the last systolic value within the $30 \mathrm{~min}$ before assuming a standing position in the morning. A $1 \mathrm{~mm} \mathrm{Hg}$ increase in the morning surge, adjusted for the 24-h systolic BP and age, was associated with a $3.3 \%$ increase $(95 \% \mathrm{CI}, 0.8-5.8 \%)$ in the risk of cardiovascular events. ${ }^{33}$

In contrast to previous studies, ${ }^{31,32}$ in the IDACO database, the morning surge in BP did not predict the stroke. It is likely that the association between stroke and the morning surge depends on the stroke subtype. ${ }^{31}$ In this study, Asians belonging to the top decile were at a significantly higher risk for hemorrhagic stroke (51 cases; HR (95\% CI), 2.28 (1.09-4.26); $P=0.03)$, but not for ischemic stroke (127 cases; HR, $1.41(0.67-2.98) ; P=0.37)$, compared with Asians with a lesser morning surge. These results were consistent with a previous report from the Ohasama study, ${ }^{31}$ but different from Kario's study, ${ }^{32}$ in which the sleep-trough morning surge significantly predicted cerebral infarcts. The different characteristics of the populations under study might explain this diversity. Indeed, Kario's study ${ }^{32}$ included older hypertensive patients (mean age, 72 years). The sleep-trough morning surge in systolic BP in the top decile $(\geqslant 55 \mathrm{~mm} \mathrm{Hg})$ was greater in Kario's study ${ }^{32}$ than in this report $(\geqslant 35 \mathrm{~mm} \mathrm{Hg}$ in Europeans and South Americans and $\geqslant 43 \mathrm{~mm} \mathrm{Hg}$ in Asians).

\section{Conclusion}

Although the morning surge in systolic pressure as well as diastolic pressure predicted the risk, we would suggest using only the rise in systolic BP in the morning as a risk indicator, because in middle-aged and older participants systolic rather than diastolic BP is the pre- 
dominant risk factor. ${ }^{36}$ Using the morning surge in BP as a risk indicator requires multiple BP readings during sleep and during the pre-awakening and awakening periods. Participants also have to complete a diary during ambulatory BP monitoring to report the sleeping and awakening periods. In our database, these two issues eliminated 4850 of 11786 available participants. Moreover, according to our recently published study in older patients with isolated systolic hypertension, ${ }^{37}$ the morning surge in $\mathrm{BP}$, irrespective of its definitions, was poorly reproducible. Nearly $30 \%$ of the participants changed their surge status either in the short term (median, 33 days) or in the long term (median, 10 months). ${ }^{37}$ These three factors might limit the clinical application of the morning surge in $\mathrm{BP}$ as a cardiovascular risk factor.

The IDACO findings established the prognostic value of the morning surge in BP in general populations. An exaggerated morning surge, exceeding the 90th percentile of the population, is an independent risk factor for mortality and cardiovascular and cardiac events. Conversely, a sleep-trough or pre-awakening morning surge in systolic BP below $20 \mathrm{~mm} \mathrm{Hg}$ is probably not associated with an increased risk of death or cardiovascular events.

\section{SHORT-TERM BP VARIABILITY}

\section{State-of-the-art before IDACO}

The predictive value of short-term reading-to-reading BP variability remains uncertain. Possible limitations of previous studies were lack of statistical power, ${ }^{38-41}$ selection of specific groups of patients, ${ }^{41-43}$ categorization of variability by arbitrary cut-off points, ${ }^{38,40,43-45}$ and sole reliance on fatal end points. ${ }^{46,47}$ Moreover, various parameters can capture short-term BP variability over $24 \mathrm{~h}$, but most studies only considered the s.d. of systolic ${ }^{40,42,48}$ or diastolic BP or both. ${ }^{44-46}$

\section{Information added by IDACO}

As measures of short-term reading-to-reading BP variability, we used $^{49}$ the s.d. over $24 \mathrm{~h}$ weighted for the time interval between consecutive readings (s.d.24), the average of the daytime and nighttime s.d.'s weighted for the duration of the daytime and nighttime interval $\left(\right.$ s.d.dn $\left._{\mathrm{dn}}\right),{ }^{50}$ and the average real variability weighted for the time interval between consecutive readings $\left(\mathrm{ARV}_{24}\right) .{ }^{40}$ The s.d.dn is the mean of day and night s.d. values corrected for the number of hours included in each of these two periods (Figure 3a), according to the
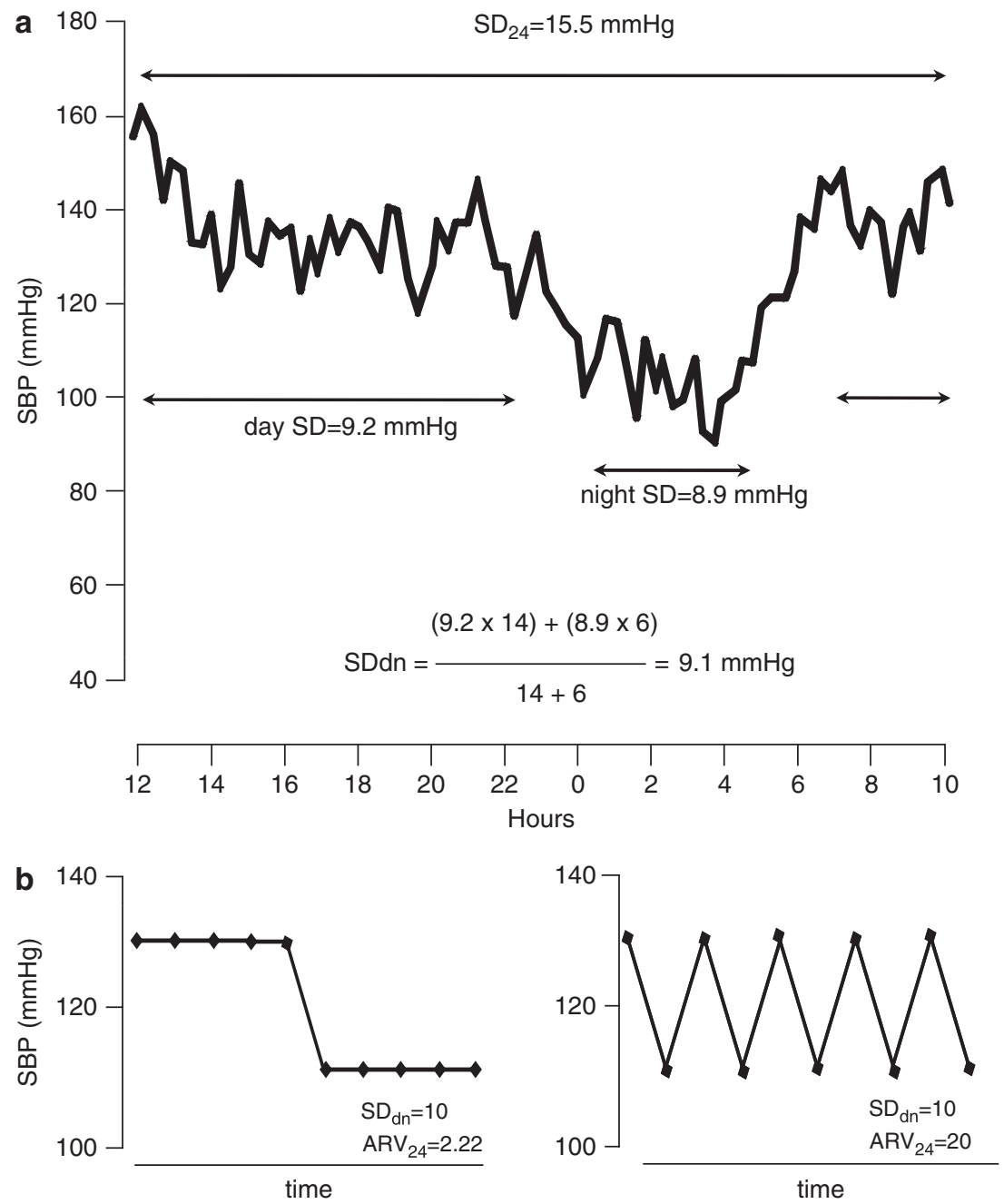

Figure 3 (a) Average of the daytime and nighttime s.d. weighted for the duration of the daytime and nighttime interval (s.d.dn), and (b) average real variability weighted for the time interval between consecutive readings $\left(\mathrm{ARV}_{24}\right)$. Reproduced with permission from Mena et al. ${ }^{40}$ and Bilo et al., ${ }^{50}$ respectively. (a) An illustrative 24-h systolic blood pressure (SBP) profile. The s.d. of the 24-h systolic BP is substantially higher than the corresponding daytime and nighttime s.d.'s, separately computed, because of the contribution of the pronounced nocturnal fall in BP. ARV 24 averages the absolute differences between consecutive readings and thereby accounts for the order of the BP readings. (b) Illustrates that for distinct BP signals, s.d. can be the same, whereas $\mathrm{ARV}_{24}$ is not. Reproduced with permission from Hansen et al. ${ }^{49}$ 
formula $^{50}$ s.d. ${ }_{\mathrm{dn}}=(($ day s.d. $\times$ hours included in the daytime $)+($ night s.d. $\times$ hours included in the nighttime))/(hours included in daytime plus nighttime). This method removes the influence of the day-night $\mathrm{BP}$ difference from the estimate of BP variability. The $\mathrm{ARV}_{24}$ averages the absolute differences of consecutive measurements and accounts in this manner for the order in which the BP measurements are obtained (Figure $3 \mathrm{~b}$ ). It is calculated by the following formula:

$$
\mathrm{ARV}=\frac{1}{\sum w} \sum_{k=1}^{n-1} w \times\left|\mathrm{BP}_{k+1}-\mathrm{BP}_{k}\right|
$$

where $k$ ranges from 1 to $N-1$ and $w$ is the time interval between $\mathrm{BP}_{k}$ and $\mathrm{BP}_{k+1} . N$ is the number of $\mathrm{BP}$ readings. The $\mathrm{ARV}_{24}$, s.d.24 and s.d.dn were highly correlated with one another; the correlation coefficients ranged from 0.75 to $0.81(P \leqslant 0.001)$ for systolic BP, and from 0.71 to $0.79(P \leqslant 0.001)$ for diastolic BP.

In adjusted models not including 24-h BP level, systolic BP variability predicted both total and cardiovascular mortality (Table $4 ; P \leqslant 0.04$ ), with the exception of s.d.24 $_{24}$ in relation to total mortality $(P=0.17)$. We obtained similar results after additional adjustment for the 24-h systolic BP, with the exception of s.d.24 and s.d.dn, which no longer predicted the cardiovascular mortality $(P \geqslant 0.71)$. Diastolic BP variability predicted the total and cardiovascular mortality both in adjusted and fully adjusted models (Table 4; $P \leqslant 0.002)$.

In adjusted analyses not including the 24-h BP level, systolic BP variability predicted all of the fatal combined with nonfatal outcomes
$(P \leqslant 0.03)$, with the exception of coronary events $(P \geqslant 0.07)$. However, in fully adjusted analyses, systolic BP variability lost its predictive value, with the exception of $\mathrm{ARV}_{24}$, in relation to all cardiovascular events combined and stroke (Table 4). Diastolic BP variability was predictive of all combined end points $(P \leqslant 0.03)$, with the exception of coronary events $(P \geqslant 0.15)$. In fully adjusted models, diastolic BP variability only predicted all cardiovascular events combined $\left(\mathrm{ARV}_{24}\right.$ and s.d.dn $)$ and fatal plus nonfatal stroke $\left(\mathrm{ARV}_{24}\right)$ (Table 4).

Figure 4 shows the absolute risk of a combined cardiovascular events in relation to 24-h BP at different levels of systolic and diastolic $\mathrm{ARV}_{24}$ ( $\mathrm{a}$ and $\mathrm{b}$ ) and in relation to $\mathrm{ARV}_{24}$ at different levels of 24-h systolic and diastolic BP (c and d). The analyses were standardized to the distributions (mean or ratio) of cohort, sex, age, 24-h heart rate, body mass index, smoking and drinking, serum cholesterol, history of cardiovascular disease, diabetes mellitus and treatment with antihypertensive drugs. Absolute risk increased with both the 24-h BP $(P<0.001)$ and $\mathrm{ARV}_{24}(P \leqslant 0.04)$. However, with the 24 -h BP in the model, $\mathrm{ARV}_{24}$ added only $0.1 \%$ to the explained risk of a composite cardiovascular event. ${ }^{49}$

\section{Interpretation}

Several prospective studies in populations ${ }^{40,46-48}$ and hypertensive patients ${ }^{38,39,41-45}$ searched for association between cardiovascular outcomes and BP variability, but reported inconsistent results. This might be due to insufficient sample size, too few events, varying definitions of the outcomes of interest or the use of different indexes of BP variability. To assess BP variability, most studies used ambulatory BP

Table 4 Multivariable-adjusted standardized hazard ratios relating outcome to blood pressure variability

\begin{tabular}{|c|c|c|c|c|c|c|}
\hline \multirow[b]{2}{*}{ Outcome (number of events) } & \multicolumn{3}{|c|}{ Systolic blood pressure } & \multicolumn{3}{|c|}{ Diastolic blood pressure } \\
\hline & s.d.24 & s.d.dn & $A R V_{24}$ & s.d. 24 & s.d.dn & $A R V_{24}$ \\
\hline s.d., $\mathrm{mm} \mathrm{Hg}$ & 15.6 & 12.2 & 11.2 & 11.8 & 9.1 & 8.5 \\
\hline \multicolumn{7}{|l|}{ Mortality } \\
\hline \multicolumn{7}{|l|}{ Total $(n=1242)$} \\
\hline Adjusted & $1.05(0.98-1.13)$ & $1.13(1.06-1.21)^{\S}$ & $1.14(1.06-1.22)^{\S}$ & $1.11(1.04-1.19)^{\S}$ & $1.17(1.09-1.24)^{\S}$ & $1.12(1.05-1.19)^{\S}$ \\
\hline Fully adjusted ${ }^{a}$ & $1.00(0.94-1.07)$ & $1.08(1.01-1.15)^{\dagger}$ & $1.11(1.04-1.18)^{\S}$ & $1.09(1.03-1.16)^{\ddagger}$ & $1.16(1.09-1.23)^{\S}$ & $1.13(1.07-1.19)^{\S}$ \\
\hline \multicolumn{7}{|l|}{ Cardiovascular $(n=487)$} \\
\hline Adjusted & $1.11(1.00-1.24)^{\dagger}$ & $1.13(1.02-1.26)^{\dagger}$ & $1.23(1.11-1.36)^{\S}$ & $1.21(1.10-1.34)^{\S}$ & $1.24(1.13-1.36)^{\S}$ & $1.27(1.17-1.38)^{\S}$ \\
\hline Fully adjusted ${ }^{a}$ & $1.03(0.93-1.13)$ & $1.05(0.95-1.17)$ & $1.17(1.07-1.28)^{\dagger}$ & $1.15(1.05-1.26)^{\ddagger}$ & $1.18(1.08-1.29)^{\S}$ & $1.21(1.12-1.31)^{\S}$ \\
\hline \multicolumn{7}{|l|}{ Fatal and nonfatal events } \\
\hline \multicolumn{7}{|l|}{ Cardiovascular $(n=1049)$} \\
\hline Adjusted & $1.13(1.06-1.22)^{\S}$ & $1.15(1.07-1.24)^{\S}$ & $1.19(1.11-1.27)^{\S}$ & $1.15(1.07-1.23)^{\S}$ & $1.16(1.09-1.24)^{\S}$ & $1.16(1.09-1.23)^{\S}$ \\
\hline Fully adjusted ${ }^{a}$ & $1.02(0.96-1.09)$ & $1.04(0.97-1.11)$ & $1.07(1.00-1.14)^{\dagger}$ & $1.05(0.99-1.12)$ & $1.07(1.01-1.14)^{\dagger}$ & $1.07(1.01-1.13)^{\dagger}$ \\
\hline \multicolumn{7}{|l|}{ Cardiac $(n=577)$} \\
\hline Adjusted & $1.13(1.02-1.24)^{\dagger}$ & $1.11(1.01-1.23)^{\dagger}$ & $1.11(1.00-1.22)^{\dagger}$ & $1.10(1.00-1.20)^{\dagger}$ & $1.11(1.02-1.21)^{\S}$ & $1.10(1.01-1.20)^{\S}$ \\
\hline Fully adjusted ${ }^{a}$ & $1.03(0.94-1.12)$ & $1.01(0.92-1.11)$ & $1.03(0.94-1.13)$ & $1.02(0.94-1.11)$ & $1.03(0.95-1.12)$ & $1.02(0.94-1.11)$ \\
\hline \multicolumn{7}{|l|}{ Coronary $(n=421)$} \\
\hline Adjusted & $1.11(0.99-1.24)$ & $1.09(0.97-1.22)$ & $1.06(0.94-1.19)$ & 1.07 (0.96-1.19) & $1.08(0.97-1.19)$ & 1.07 (0.97-1.19) \\
\hline Fully adjusted ${ }^{a}$ & $1.07(0.96-1.18)$ & $1.04(0.93-1.16)$ & $1.03(0.93-1.14)$ & $1.02(0.93-1.13)$ & $1.02(0.92-1.12)$ & $1.02(0.92-1.12)$ \\
\hline \multicolumn{7}{|l|}{ Stroke $(n=457)$} \\
\hline Adjusted & $1.13(1.01-1.26)^{\dagger}$ & $1.16(1.04-1.30)^{\dagger}$ & $1.25(1.13-1.39)^{\S}$ & $1.22(1.13-1.35)^{\S}$ & $1.22(1.10-1.35)^{\S}$ & $1.26(1.14-1.38)^{\S}$ \\
\hline Fully adjusted ${ }^{a}$ & $0.98(0.88-1.09)$ & $1.03(0.92-1.14)$ & $1.10(1.00-1.21)^{\dagger}$ & 1.08 (0.99-1.19) & 1.09 (0.99-1.20) & $1.14(1.05-1.23)^{\dagger}$ \\
\hline
\end{tabular}

Abbreviation: ARV, average real variability over $24 \mathrm{~h}$.

aAdditionally adjusted for the corresponding 24 -h blood pressure level.

Values are standardized hazard ratios ( $95 \%$ confidence intervals), which express the risk per s.d. increase in the predictor variables. All hazard ratios were computed by Cox regression stratified for cohort and adjusted for sex, age, 24-h heart rate, body mass index, smoking and drinking, serum cholesterol, history of cardiovascular disease, diabetes mellitus and treatment with antihypertensive drugs.

Significance of the hazard ratios: ${ }^{\dagger} P<0.05,{ }^{\ddagger} P<0.01$ and ${ }^{\S} P<0.001$. Reproduced with permission from Hansen et al. ${ }^{49}$ 

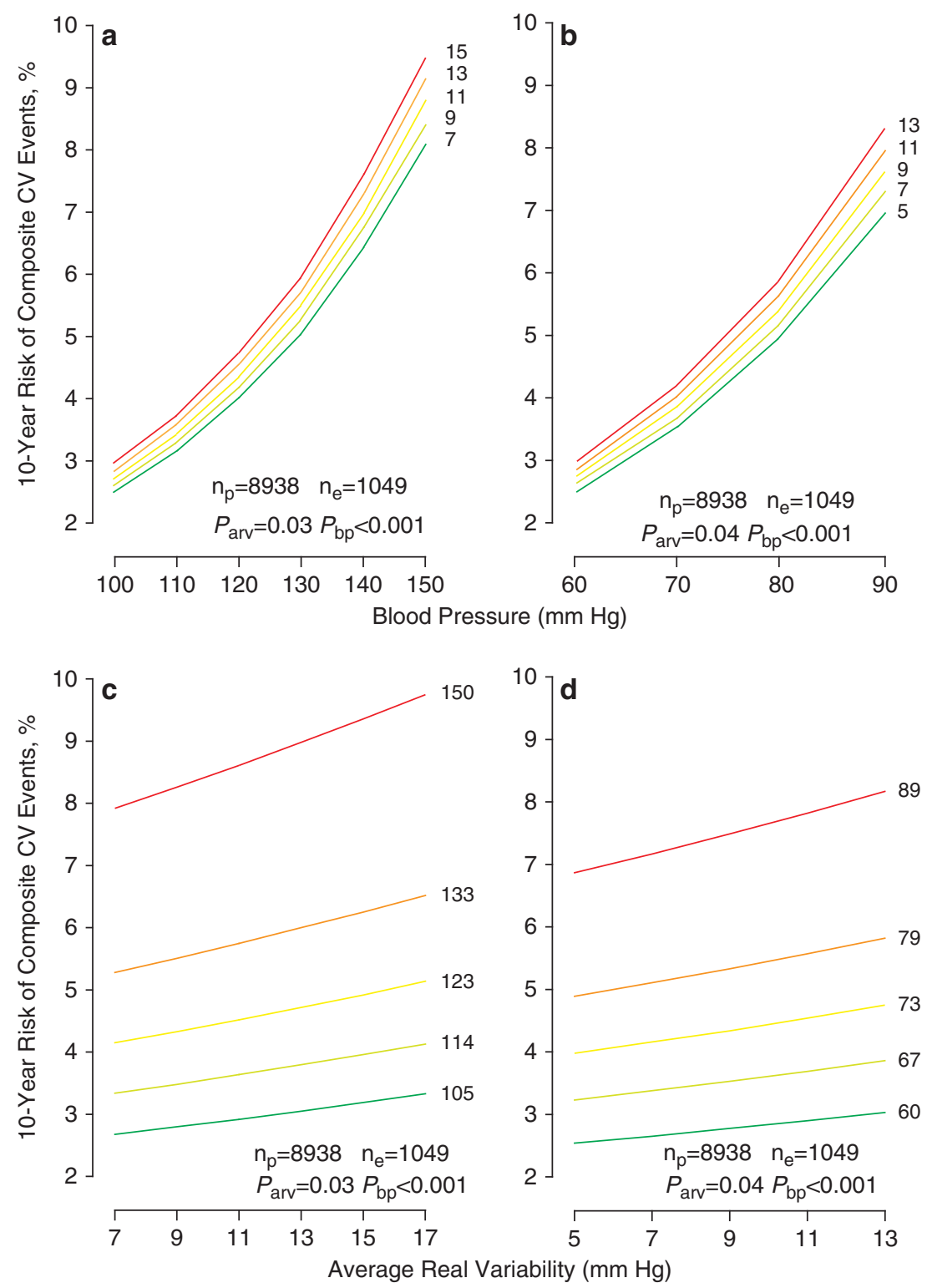

Figure 4 Ten-year absolute risk of combined cardiovascular (CV) events in relation to 24-h BP (a, b) at different levels of systolic and diastolic average real variability over $24 \mathrm{~h}\left(\mathrm{ARV}_{24}\right)$ and in relation to $\mathrm{ARV}_{24}(\mathbf{c}, \mathrm{d})$ at different levels of 24 -h systolic and diastolic blood pressure (BP). The analyses were standardized to the distributions (mean or ratio) of cohort, sex, age, 24-h heart rate, body mass index, smoking and drinking, serum cholesterol, history of cardiovascular disease, diabetes mellitus and treatment with antihypertensive drugs. Plotted values of 24-h BP (a, b) and ARV (c, d) span the 5th to 95th percentile interval. The continuous risk functions correspond to the 5th, 25th, 50th, 75th and 95th percentiles of ARV (a, b) and 24-h BP (c, d). P-values are for the independent effect of ARV $\left(P_{\text {arv }}\right)$ and 24-h BP $\left(P_{\mathrm{bp}}\right)$. np and ne indicate the number of participants at risk and the number of cardiovascular events. Reproduced with permission from Hansen et al. ${ }^{49}$

monitoring with intermittent readings at intervals ranging from $15^{45}$ to $30^{41} \mathrm{~min}$ throughout $24 \mathrm{~h}$. In the Northwick Park study, ${ }^{39}$ the investigators carried out continuous intra-arterial recordings, but did not fully exploit the potential of this recording technique. Instead of analyzing variability in the frequency domain, they computed hourly means of BP and the within-participant s.d. of the hourly means as a measure of each participant's BP variability. In the Ohasama study, investigators used the self-measured $\mathrm{BP}^{51}$ in addition to ambulatory BP monitoring. ${ }^{46}$ In all but two studies, ${ }^{40,43}$ the researchers used the s.d. of daytime, nighttime or 24-h BP as indexes of variability. Four studies $^{41,44-46}$ deliberately did not report on the predictive value of the variability in the $24-\mathrm{h} \mathrm{BP}$, because the diurnal BP profile also includes long-term variability, which is captured by the night-to-day BP ratio. To address this potential concern, we computed s.d.dn and $\mathrm{ARV}_{24}$ as measures of variability. Only two other prospective studies, one in a small general Venezuelan population (312 participants with 31 composite cardiovascular end points), ${ }^{40}$ and one in a hypertensive population, ${ }^{43}$ implemented $\mathrm{ARV}_{24}$. Bilo et al. ${ }^{50}$ were the first to propose s.d.dn.

From a clinical point of view, the IDACO findings suggest that, although statistically significant, the clinical applicability of BP variability for risk stratification might be limited. First, antihypertensive drug treatment is bound to influence BP variability. Second, the reproducibility of BP variability is poor. In 97 normotensive 
participants ${ }^{52}$ the relative repeatability coefficient of the s.d. of the 24-h BP, expressed as a percentage of the 5th to 95th percentile interval, was $13 \%$ systolic and $16 \%$ diastolic, whereas for the $24-\mathrm{h}$ BP these coefficients were 4 and 5\%, respectively, lower values indicating better reproducibility. ${ }^{52}$ Finally, the added value in terms of absolute risk was modest in our population. For example, in adjusted analyses (Figure 4), the increase in the 10-year absolute risk of a composite cardiovascular event associated with an increase from the median to the 75 th percentile was $0.21 \%$ for systolic $\mathrm{ARV}_{24}(1.5 \mathrm{~mm} \mathrm{Hg})$ and $1.23 \%$ for the 24 -h systolic BP $(9.8 \mathrm{~mm} \mathrm{Hg})$. The corresponding estimates for diastolic BP were $0.16 \%(2.3 \mathrm{~mm} \mathrm{Hg})$ and $1.05 \%$ $(5.8 \mathrm{~mm} \mathrm{Hg})$, respectively.

\section{Conclusion}

The IDACO report established that short-term reading-to-reading BP variability is an independent risk factor, but, moreover, it also highlighted that the level of the 24-h BP remains the primary BP-related risk factor to account for in clinical practice. For research making use of intermittent techniques of ambulatory BP monitoring, our current findings suggest that both s.d.dn and $\mathrm{ARV}_{24}$ might be useful measures, but not the s.d. computed over the whole day, which also includes the day-night BP difference.

\section{PERSPECTIVES}

Thus, the IDACO observations support the concept that the ambulatory BP should be recorded over the whole day, as both the nighttime and daytime BP levels carry prognostic information. Moreover, the level of the 24-h BP remains the primary blood pressurerelated risk factor to account for in clinical practice. Notwithstanding the new insights generated by the IDACO, several research questions about the prognostic value of BP variability remain to be answered in the future. First, chronotherapy ${ }^{53}$ means timing the administration of antihypertensive drugs in such a way that the BP is lowered over $24 \mathrm{~h}$, while a normal night-to-day BP ratio is preserved. However, there is no evidence supporting the efficacy of chronotherapy in terms of BP control $^{54}$ or outcome. ${ }^{26}$ Second, the morning surge above the 90th percentile significantly and independently predicted the cardiovascular outcome and might contribute to risk stratification by ambulatory BP monitoring. However, randomized clinical trials are needed to answer the question whether restoring the diurnal BP profile might be beneficial in terms of prevention of cardiovascular morbidity and mortality. Finally, the value of BP variability in comparison with the 24-h BP level seems limited. However, in the setting of clinical research, studies of BP variability will continue to generate meaningful information.

\section{ACKNOWLEDGEMENTS}

The European Union (grants IC15-CT98-0329-EPOGH, LSHM-CT-2006037093 and HEALTH-F4-2007-201550), the Fonds voor Wetenschappelijk Onderzoek Vlaanderen (Ministry of the Flemish Community, Brussels, Belgium; grants G.0575.06 and G.0734.09) and the Katholieke Universiteit Leuven (grants OT/00/25 and OT/05/49) gave support to the Studies Coordinating Centre in Leuven. The European Union (grants LSHM-CT-2006-037093 and HEALTH-F4-2007-201550) also supported the research groups in Kraków and Shanghai. We thank Ms Sandra Covens and Ms Ya Zhu (Studies Coordinating Centre, University of Leuven, Leuven, Belgium) for their secretarial assistance.

1 O'Brien E, Asmar R, Beilin L, Imai Y, Mancia G, Mengden T, Myers M, Padfield P, Palatini P, Parati G, Pickering T, Redon J, Staessen J, Stergiou G, Verdecchia P, on behalf of the European Society of Hypertension Working Group on Blood
Pressure Monitoring. Practice guidelines of the European Society of Hypertension for clinic, ambulatory and self blood pressure measurement. J Hypertens 2005; 23 : 697-701.

2 Staessen JA, Wang J, Bianchi G, Birkenhäger WH. Essential hypertension. Lancet 2003; 361: 1629-1641.

3 Prospective Studies Collaboration. Age-specific relevance of usual blood pressure to vascular mortality: a meta-analysis of individual data for one million adults in 61 prospective studies. Lancet 2002; 360: 1903-1913.

4 Asia Pacific Cohort Studies Collaboration. Blood pressure and cardiovascular disease in the Asia Pacific region. J Hypertens 2003; 21: 707-716.

5 Staessen JA, O'Brien ET, Amery AK, Atkins N, Baumgart P, De Cort P, Degaute JP, Dolenc P, De Gaudemaris R, Enström I, Fagard R, Gosse P, Gourlay S, Hayashi H, Imai Y, James G, Kawasaki T, Kuschnir E, Kuwajima I, Lindholm L, Liu L, Macor F, Mancia G, McGrath B, Middeke M, Ming J, Omboni S, Otsuka K, Palatini P, Parati G, Pieper C, Verdecchia P, Zachariah P, Zhang W. Ambulatory blood pressure in normotensive and hypertensive subjects: results from an international database. J Hypertens 1994; 12(Suppl 7): S1-S12.

6 Li Y, Staessen JA, Lu L, Li LH, Wang GL, Wang JG. Is isolated nocturnal hypertension a novel clinical entity? Findings from a Chinese population study. Hypertension 2007; 50: 333-339.

7 Staessen JA, Bieniaszewski L, O'Brien E, Gosse P, Hayashi H, Imai Y, Kawasaki T, Otsuka K, Palatini P, Thijs L, Fagard R, on behalf of the 'Ad Hoc ' Working Group. Nocturnal blood pressure fall on ambulatory monitoring in a large international database. Hypertension 1997; 29: 30-39.

8 Hansen TW, Jeppesen J, Rasmussen F, Ibsen H, Torp-Pedersen C. Ambulatory blood pressure monitoring and mortality: a population-based study. Hypertension 2005; 45: 499-504.

9 Staessen JA, Bieniaszewski L, O'Brien ET, Imai Y, Fagard R. An epidemiological approach to ambulatory blood pressure monitoring: the Belgian population study. Blood Press Monit 1996; 1: 13-26.

10 Kuznetsova T, Malyutina S, Pello E, Thijs L, Nikitin Y, Staessen JA. Ambulatory blood pressure of adults in Novosibirsk, Russia: interim report on a population study. Blood Press Monit 2000; 5: 291-296.

11 Kuznetsova T, Staessen JA, Kawecka-Jaszcz K, Babeanu S, Casiglia E, Filipovský J, Nachev C, Nikitin Y, Peleská J, O'Brien E, on behalf of the EPOGH Investigators. Quality control of the blood pressure phenotype in the European Project on Genes in Hypertension. Blood Press Monit 2002; 7: 215-224.

12 Ohkubo T, Hozawa A, Yamaguchi J, Kikuya M, Ohmori K, Michimata M, Matsubara M, Hashimoto J, Hoshi H, Araki T, Tsuji I, Satoh H, Hisamichi S, Imai Y. Prognostic significance of the nocturnal decline in blood pressure in individuals with and without high 24-h blood pressure: the Ohasama study. J Hypertens 2002; 20: 2183-2189.

13 Schettini C, Bianchi M, Nieto F, Sandoya E, Senra H, Hypertension Working Group. Ambulatory blood pressure. Normality and comparison with other measurements. Hypertension 1999; 34 (Part 2): 818-825.

14 O'Brien E, Sheridan J, O'Malley K. Dippers and non-dippers. Lancet 1988; ii: 397.

15 Ingelsson E, Björklund K, Lind L, Ärnlöv J, Sundström J. Diurnal blood pressure pattern and risk of congestive heart failure. JAMA 2006; 295: 2859-2866.

16 Mancia G, Facchetti R, Bombelli M, Grassi G, Sega R. Long-term risk of mortality associated with selective and combined elevation in office, home, and ambulatory blood pressure. Hypertension 2006; 47: 846-853.

17 Verdecchia P, Porcellati C, Schillaci G, Borgioni C, Ciucci A, Battistelli M, Guerrieri M, Gatteschi C, Zampi I, Santucci A, Santucci C, Reboldi G. Ambulatory blood pressure. An independent predictor of prognosis in essential hypertension. Hypertension 1994; 24: 793-801.

18 Staessen JA, Thijs L, Fagard R, O’Brien ET, Clement D, de Leeuw PW, Mancia G, Nachev C, Palatini P, Parati G, Tuomilehto J, Webster J, for the Systolic Hypertension in Europe Trial Investigators. Predicting cardiovascular risk using conventional vs ambulatory blood pressure in older patients with systolic hypertension. JAMA 1999; 282: 539-546.

19 Kario K, Pickering TG, Matsuo T, Hoshide S, Schwartz JE, Shimada K. Stroke prognosis and abnormal nocturnal blood pressure falls in older hypertensives. Hypertension 2001; 38: 852-857.

20 Clement DL, De Buyzere ML, De Bacquer DA, de Leeuw PW, Duprez DA, Fagard RH, Gheeraert PJ, Missault LH, Braun JJ, Six RO, Van der Niepen P, O'Brien E, for the Office versus Ambulatory Pressure Study investigators. Prognostic value of ambulatory blood-pressure recordings in patients with treated hypertension. N Engl J Med 2003; 348: 2407-2415.

21 Dolan E, Stanton A, Thijs L, Hinedi K, Atkins N, McClory S, Den Hond E, McCormack P, Staessen JA, O'Brien E. Superiority of ambulatory over clinic blood pressure measurement in predicting mortality. The Dublin Outcome Study. Hypertension 2005; 46: 156-161.

22 Ben-Dov IZ, Kark JD, Ben-Ishay D, Mekler J, Ben-Arie L, Bursztyn M. Predictors of allcause mortality in clinical ambulatory monitoring. Unique aspects of blood pressure during sleep. Hypertension 2007; 49: 1235-1241.

23 Schwartz GL, Bailey KR, Mosley T, Knopman DS, Jack Jr CR, Canzanello VJ, Turner ST. Association of ambulatory blood pressure with ischemic brain injury. Hypertension 2007; 49: 1228-1234.

24 Boggia J, Li Y, Thijs L, Hansen TW, Kikuya M, Björklund-Bodegård K, Richart T, Ohkubo T, Kuznetsova T, Torp-Pedersen C, Lind L, Ibsen H, Imai Y, Wang JG, Sandoya E, O'Brien E, Staessen JA, on behalf of the International Database on Ambulatory blood pressure monitoring in relation to Cardiovascular Outcomes (IDACO) investigators. Prognostic accuracy of day versus night ambulatory blood pressure: a cohort study. Lancet 2007; 370: 1219-1229. 
25 Kikuya M, Hansen TW, Thijs L, Björklund-Bodegård K, Kuznetsova T, Ohkubo T, Richart T, Torp-Pedersen C, Lind L, Ibsen H, Imai Y, Staessen JA, and on behalf of the IDACO Investigators. Diagnostic thresholds for ambulatory blood pressure monitoring based on 10-year cardiovascular risk. Blood Press Monit 2007; 12: 393-395.

26 Omboni S, Parati G, Palatini P, Vanasia A, Muiesan ML, Cuspidi C, Mancia G. Reproducibility and clinical value of nocturnal hypotension: prospective evidence from the SAMPLE study. J Hypertens 1998; 16: 733-738.

27 Mochizuki Y, Okutani M, Donfeng Y, Iwasaki H, Takusagawa M, Kohno I, Mochizuki S, Umetani K, Ishii H, Ijiri H, Komori S, Tamura K. Limited reproducibility of circadian variation in blood pressure dippers and nondippers. Am J Hypertens 1998; 11: 403-409.

28 Muller JE, Stone PH, Turi ZG, Rutherford JD, Czeisler CA, Parker C, Poole WK, Passamani E, Roberts R, Robertson T, Sobel BE, Willerson JT, Braunwald E, and the MILIS Study Group. Circadian variation in the frequency of onset of acute myocardial infarction. N Engl J Med 1985; 313: 1315-1322.

29 Tofler GH, Muller JE, Stone PH, Forman S, Solomon RE, Knatterud GL, Braunwald E. Modifiers of timing and possible triggers of acute myocardial infarction in the Thrombolysis in Myocardial Infarction Phase II (TIMI II) Study Group. J Am Coll Cardiol 1992; 20: 1049-1055.

30 Shimada K, Kario K, Umeda Y, Hoshide S, Hoshide Y, Eguchi K. Early morning surge in blood pressure. Blood Press Monit 2001; 2001: 349-353.

31 Metoki H, Ohkubo T, Asayama K, Obara T, Hashimoto J, Totsune K, Hoshi H, Satoh H, Imai K. Prognostic significance for stroke of a morning pressor surge and a nocturnal blood pressure decline: The Ohasama Study. Hypertension 2006; 47: 149-154.

32 Kario K, Pickering TG, Umeda Y, Hoshide S, Hoshide Y, Morinari M, Murata M, Kuroda T, Schwartz JE, Shimada K. Morning surge in blood pressure as predictor of silent and clinical cerebrovascular disease in elderly hypertensives. A prospective study. Circulation 2003; 107: 1401-1406.

33 Gosse P, Lasserre R, Minifie C, Lemetayer P, Clementy J. Blood pressure surge on rising. J Hypertens 2004; 22: 1113-1118.

34 Ohkubo T, Metoki H, Imai Y. Prognostic significance of morning surge in blood pressure: which definition, which outcome? Blood Press Monit 2008; 13: 161-162.

35 Li Y, Hansen TW, Kikuya M, Boggia J, Richart T, Metoki H, Ohkubo T, Torp-Pedersen C, Kuznetsova T, Stolarz-Skrzypek K, Tikhonoff V, Malyutina S, Casiglia E, Nikitin Y, Sandoya E, Kawecka-Jaszcz K, Ibsen H, Imai Y, Wang JG, Staessen JA, on behalf of the International Database on Ambulatory blood pressure monitoring in relation to Cardiovascular Outcomes (IDACO) investigators. Prognostic value of the morning blood pressure surge in 5645 subjects from 8 populations. Hypertension 2010; 55: 1049-1057.

36 Franklin SS, Larson MG, Khan SA, Wong ND, Leip EP, Kannel WB, Levy D. Does the relation of blood pressure to coronary heart disease change with aging? The Framingham Heart Study. Circulation 2001; 103: 1245-1249.

37 Wizner B, Dechering DG, Thijs L, Atkins N, Fagard R, O'Brien E, de Leeuw PW, Parati G, Palatini P, Clement D, Grodzicki T, Kario K, Staessen JA. Short-term and long-term reproducibility of the morning blood pressure in older patients with isolated systolic hypertension. J Hypertens 2008; 26: 1328-1335.

38 Eto M, Akishita M, Kozaki K, Watanabe T, Kim S, Hashimoto M, Ako J, lijima K, Sudoh $\mathrm{N}$, Yoshizumi M, Oucji Y. Impact of blood pressure variability on cardiovascular events in elderly patients with hypertension. Hypertens Res 2005; 28: 1-7.

39 Khattar RS, Swales JD, Dore C, Senior R, Lahiri A. Effect of aging on the prognostic significance of ambulatory systolic, diastolic, and pulse pressure in essential hypertension. Circulation 2001; 104: 783-789.
40 Mena L, Pintos S, Queipo NV, Aizpúrua JA, Maestre G, Sulbarán T. A reliable index for the prognostic significance of blood pressure variability. J Hypertens 2003; 23: 505-511.

41 Eguchi K, Ishikawa J, Hoshide S, Pickering TG, Schwartz JE, Shimada K, Kario K. Nighttime blood pressure variability is a strong predictor for cardiovascular events in patients with Type 2 diabetes. Am J Hypertens 2009; 22: 46-51.

42 Pringle E, Phillips C, Thijs L, Davidson C, Staessen JA, de Leeuw PW, Jaaskivi M, Nachev C, Parati G, O'Brien ET, Tuomilehto J, Webster J, Bulpitt CJ, Fagard RH, on behalf of the Syst-Eur Investigators. Systolic blood pressure variability as a risk factor for stroke and cardiovascular mortality in the elderly hypertensive population. J Hypertens 2003; 21: 2251-2257.

43 Pierdomenico SD, Nicola MD, Esposito AL, Mascio RD, Ballone E, Lapenna D, Cuccurullo F. Prognostic value of different indices of blood pressure variability in hypertensive patients. Am J Hypertens 2009; 22: 842-847.

44 Pierdomenico SD, Lapenna D, Di Tommaso R, Di Carlo S, Esposito AL, Di Mascio R, Ballone E, Cuccurullo F, Mezzetti A. Blood pressure variability and cardiovascular risk in treated hypertensive patients. Am J Hypertens 2006; 19: 991-997.

45 Verdecchia P, Angeli F, Gattobigio R, Rapicetta C, Reboldi G. Impact of blood pressure variability on cardiac and cerebrovascular complications in hypertension. Am J Hypertens 2007; 20: 154-161.

46 Kikuya M, Hozawa A, Ohokubo T, Tsuji I, Michimata M, Matsubara M, Ota M, Nagai K, Araki T, Satoh H, Ito S, Hisamichi S, Imai Y. Prognostic significance of blood pressure and heart rate variabilities. The Ohasama Study. Hypertension 2000; 36: 901-906.

47 Mancia G, Bombelli M, Facchetti R, Madotto F, Corrao G, Trevano FQ, Grassi G, Sega R. Long-term prognostic value of blood pressure variability in the general population. Results of the Pressioni Arteriose Monitorate a Loro Associazioni study. Hypertension 2007; 49: 1265-1270.

48 Björklund K, Lind L, Zethelius B, Berglund L, Lithell H. Prognostic significance of 24-h ambulatory blood pressure characteristics for cardiovascular morbidity in a population of elderly men. J Hypertens 2004; 22: 1691-1697.

49 Hansen TW, Thijs L, Li Y, Boggia J, Kikuya M, Bjorklund-Bodegard K, Richart T, Ohkubo T, Jeppesen J, Torp-Pedersen C, Dolan E, Kuznetsova T, Stolarz-Skrzypek K, Tikhonoff V, Malyutina S, Casiglia E, Nikitin Y, Lind L, Sandoya E, Kawecka-Jaszcz K, Imai Y, Wang J, Ibsen H, O'Brien E, Staessen JA. Prognostic value of reading-to-reading blood pressure variability over $24 \mathrm{~h}$ in 8938 subjects from 11 populations. Hypertension 2010; 55: 1040-1048.

50 Bilo G, Giglio A, Styczkiewicz K, Caldara G, Maronati A, Kawecka-Jaszcz K, Mancia G, Parati G. A new method for assessing 24-h blood pressure variability after excluding the contribution of nocturnal blood pressure fall. J Hypertens 2007; 25: 2058-2066.

51 Kikuya M, Ohkubo T, Metoki H, Asayama K, Hara H, Obara T, Inoue R, Hoshi H, Hashimoto J, Totsune K, Satoh H, Imai K. Day-by-day variability of blood pressure and heart rate at home as a novel predictor of prognosis The Ohasama Study. Hypertension 2008; 52: 1045-1050.

52 Thijs L, Staessen J, Fagard R, Zachariah P, Amery A. Number of measurements required for the analysis of diurnal blood pressure profile. J Hum Hypertens 1994; 8: 239-244.

53 Scarpelli PT, Gallo M, Chiari G. Chronobiology of blood pressure. J Nephrol 2000; 13: 197-204.

54 Hermida RC, Calvo C, Ayala DE, Dominguez MJ, Covelo M, Fernández JR, Mojón A, López JE. Administration time-dependent effects of valsartan on ambulatory blood pressure in hypertensive subjects. Hypertension 2003; 42: 283-290.

55 O'Brien E, Murphy J, Tyndall A, Atkins N, Mee F, McCarthy G, Staessen J, Cox J, O'Malley K. Twenty-four-hour ambulatory blood pressure in men and women aged 17-80 years: the Allied Irish Bank Study. J Hypertens 1991; 9: 355-360. 